STRUCTURAL BIOLOGY COMMUNICATIONS

ISSN 2053-230X

Keywords: crystallization; innovation.

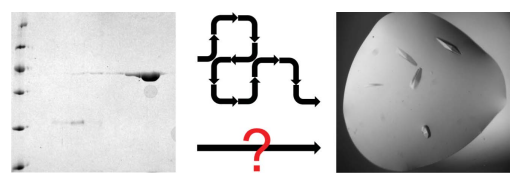

(C) 2020 International Union of Crystallography

\section{Innovation versus practice in biological crystallization}

\author{
Janet Newman $^{\mathrm{a} *}$ and Mark J. van Raaij ${ }^{\mathrm{b} *}$ \\ ${ }^{a}$ Collaborative Crystallisation Centre (C3), CSIRO, 343 Royal Parade, Parkville, VIC 3052 Australia, and ${ }^{\mathbf{b}}$ Department of \\ Molecular Structure, Centro Nacional de Biotecnologia, Consejo Superior de Investigaciones Cientificas, Madrid, \\ E-28049, Spain.*Correspondence e-mail: janet.newman@csiro.au,mjvanraaij@cnb.csic.es
}

Edward Snell, of the Hauptmann Woodward Institute in Buffalo, NY has a great introduction to his talks on crystallization

Without crystals, there is no crystallography - without crystallography, there are no crystallographers.

This succinctly points out the direct relationship between crystal growth and our professional livelihood. And yet, despite that, there is little concerted effort at getting better at growing well diffracting crystals. Looking back over the last 50 years of macromolecular crystal growth, there have been arguably only four game changers - the introduction of polyethylene glycols (PEGs) as precipitants (1970s) (McPherson, 1976); the introduction of pre-mixed crystallization screens (1990s) (Jancarik \& Kim, 1991); the commercialization of automation (2000s) (Shaw Stewart \& Mueller-Dieckmann, 2014) and the widespread adoption of matrix seeding (2000s) (D'Arcy et al., 2014).

The process of crystal growth is fraught with uncertainty and requires large amounts of sample and trials - it thus encourages reliance on tried conditions and standard setups. The common feature of the factors mentioned above is their ease of implementation, for example PEGs were widely available and their use required no changes to the current technologies; the Jancarik and Kim screen and its brethren made it so much easier to start a novel project; automation was not widely adopted until it was easier than manual setups and matrix seeding fits seamlessly into our modern workflow.

What is it about crystallization that makes it so hard to improve? There are a number of reasons: the samples that go into crystallization are very diverse - from monomeric proteins to RNA haptomers to multi-subunit complexes and a lot of in-betweens. Properties such as the solubility and monodispersity of the sample do relate to crystallizability (Borgstahl, 2007), but offer little guidance as to how to actually get the sample to crystallize. Much of structural biology is carried out in academia, where the human resources for experimental work are often students. As sample preparation and crystallization come first in a structural biology project, these tasks are tackled by students at the beginning of their tenure, when they have the least experience, and are more likely to rely on techniques easily available in their institution. And even more fundamental is that the goal of most crystallization programs is not to create new and better crystallization technologies, but to understand the biology of the system under investigation.

In summary, we still don't know what factors make a given sample crystallize and continued innovation is necessary. Acta Cryst. $F$ is committed to bringing these new crystallization developments and innovations to the attention of a wider audience. The current issue has a nice example of crystallization innovation in the paper by the Skerra group on the suitability of PAS-type molecules as crystallization agents (Schiefner et al., 2020). PAS-type molecules are low-complexity proteins consisting of only two or three types of amino acids, in a chain of defined sequence and length. The solution properties of these macromolecules resemble mid-weight PEGs, and indeed, they have been shown to successfully replace PEG 3350 for two proteins. Previous crystallization innovations, like lipidic cubic phases for membrane proteins (Landau \& Rosenbusch, 1996), imprinted hydrogel nucleants (Saridakis et al., 2011), lattices of maltose binding protein (Waugh, 2016) and other crystal-forming macromolecules have been very useful, but perhaps been less widely applicable than hoped. The innovation presented by Schiefner $e t$ al. adds to 
the available set of protein precipitants and the future will tell how broadly applicable it will be and how widely it will be taken up by other researchers.

Crystallization breakthroughs require both innovation and accessibility, both of which require significant resources. However, even when crystals are obtained, they do not guarantee our desired endpoint, which is to understand a biological system. X-ray data need to be coaxed from the crystals, structures coaxed from these data and insight coaxed from the structures. There doesn't seem to be a problem investing resources where there is a shorter or more assured path between sample and biological insight - the billions spent recently on high-end electron microscopes attest to this. Who knows what crystallization experts could do given these kinds of resources? Perhaps crystallography labs in the future would have access to integrated systems that analyse initial screenbased trials, improve them autonomously and obtain well diffracting crystals - all with minimal human intervention.

In the meantime, Acta Cryst. F continues to be dedicated to helping to improve the crystallization process by publishing new discoveries, from small tricks and tips to modern innovative techniques. We welcome articles that advance our understanding of any aspect of the crystallization process. The current situation with the COVID-19 pandemic has meant that many new discoveries are being made and any articles associated with these are especially welcome.

\section{References}

Borgstahl, G. E. (2007). Methods Mol. Biol. 363, 109-129.

D’Arcy, A., Bergfors, T., Cowan-Jacob, S. W. \& Marsh, M. (2014). Acta Cryst. F70, 1117-1126.

Jancarik, J. \& Kim, S.-H. (1991). J. Appl. Cryst. 24, 409-411.

Landau, E. M. \& Rosenbusch, J. P. (1996). Proc. Natl Acad. Sci. 93, 14532-14535.

McPherson, A. (1976). J. Biol. Chem. 251, 6300-6303.

Saridakis, E., Khurshik, S., Govada, L., Phan, Q., Hawkins, D., Crichlow, G. V., Lolis, E., Reddy, S. M. \& Chayen, N. E. (2011). Proc. Natl Acad. Sci. 108, 11081-11086.

Schiefner, A., Walser, R., Gebauer, M. \& Skerra, A. (2020). Acta Cryst. F76, 320-325.

Shaw Stewart, P. \& Mueller-Dieckmann, J. (2014). Acta Cryst. F70, 686-696.

Waugh, D. S. (2016). Prot. Sci. 25, 559-571. 